\title{
ANALYSIS OF THE ASSOCIATED STRESS DISTRIBUTIONS TO THE NONLINEAR FORCED VIBRATIONS OF FUNCTIONALLY GRADED MULTI-CRACKED BEAMS
}

\author{
Mohcine CHAJDI ${ }^{1}$, Ahmed ADRI ${ }^{2}$, Khalid EL BIKRI ${ }^{1}$, Rhali BENAMAR ${ }^{3}$ \\ ${ }^{1}$ Mohammed V University in Rabat, ENSAM - Rabat, MSSM, B.P.6207, Rabat Instituts, Rabat, Morocco, \\ mohcine.chajdi@um5s.net.ma, k.elbikri@um5s.net.ma \\ ${ }^{2}$ Hassan II University of Casablanca, EST - Casablanca, LMPGI, B.P.8012, Oasis Casablanca, Morocco, \\ ahmedadri@gmail.com \\ ${ }^{3}$ Mohammed V University in Rabat, EMI - Rabat, LERSIM, B.P.765, Agdal, Rabat, Morocco, \\ rhali.benamar@gmail.com
}

\section{Abstract}

Geometrically non-linear vibrations of functionally graded Euler-Bernoulli beams with multi-cracks, subjected to a harmonic distributed force, are examined in this paper using a theoretical model based on Hamilton's principle and spectral analysis. The homogenisation procedure is performed, based on the neutral surface approach, and reduces the FG beams analysis to that of an equivalent homogeneous multi-cracked beam. The so-called multidimensional Duffing equation obtained and solved using a simplified method (second formulation) previously applied to various non-linear structural vibration problems. The curvature distributions associated to the multi-cracked beam forced deflection shapes are obtained for each value of the excitation level and frequency. The parametric study performed in the case of a beam and the detailed numerical results are given in hand to demonstrate the effectiveness of the proposed procedure, and in the other hand conducted to analyse many effects such as the beam material property, the presence of crack, the vibration amplitudes and the applied harmonic force on the non-linear dynamic behaviour of FG beams.

Keywords: nonlinear vibration; multi-cracks; functionally graded beams; forced vibrations

\section{INTRODUCTION}

Structures made of functionally graded materials (FGM) have a very wide area of industrial applications, especially those involving severe environments with high temperatures or important temperature gradients, due to the properties of this type of materials characterized by a smooth and a continuous transition in the compositional profile. However, damages in the form of cracks may be observed in these materials during their operational life as a result of various external or internal influences or even during the manufacturing process. It is known that a crack in a structure produces a local change in the stiffness altering its dynamic characteristics and increasing its vulnerability. It is therefore of a crucial importance to understand and control the structural behaviour in such situations in order to ensure the safety and durability of the structural components. Structural health surveillance and vibration analysis are the most often used techniques by researchers and engineers in order to maintain the structural integrity. As beams are very currently used as structural components, the investigation of the dynamic behaviour of functionally graded cracked beams has been an active area of research in the last few years. Numerous methods, based on analytical, semi- analytical and numerical techniques, are available in the open literature, to investigate the effects of cracks on the linear and non-linear vibration characteristics in both the free and forced regimes. Sridhar et al. used a pseudo-spectral finite element method to analyse the wave propagation in functionally graded beam (FGB) and layered structures with and without vertical or horizontal cracks [1]. Yang and Chen analytically studied the influence of an open edge crack on the free vibration and elastic buckling of FGB using the EulerBernoulli beam theory and the rotational spring model with different boundary conditions [2]. Later, $\mathrm{Ke}$ et al. studied the free vibration and elastic buckling of cracked FGB with different end conditions within the Timoshenko beam theory using the same method as that of Yang and Chen [3]. Matbuly et al. used the differential quadrature method to study the free vibration of a FG cracked Timoshenko beam resting on a Winkler-Pasternak foundation [4]. Yu and Chu used the p-version of the finite element method to study the transverse vibration characteristics of cracked FGB [5]. Ferezqi et al. presented an analytical investigation of the free vibrations of a FG cracked Timoshenko beam employing a wave approach [6]. Lien et al. used [7] an alternative approach called dynamic stiffness method to establish a new form of frequency 
equation of free vibration multi-cracked FG Timoshenko beams taking into account the actual position of neutral plane. Shabani and Cunedioglu [8] investigated the free vibrations of a cantilever symmetric FG sandwich Timoshenko beam with two cracks using the finite element method. Very recently, Kou and Yang developed a mesh-free boundary-domain integral equation method to analyse the free vibration behaviours of the FGB with edged cracks [9]. On the other hand, many researches have examined the case of a cracked FGB subjected to external loadings in the form of moving loads or axial forces [10-16]. Lately, Lin-Feng Zhu et al. [17] analysed the vibrational power flow of a cracked FG Timoshenko beam under a concentrated transverse harmonic force using the wave propagation approach to obtain the input power flow and transmitted power flow and applying the continuous wavelet transform to deal with the inverse problem of crack identification. It should be pointed out that all of the aforementioned investigations were limited to linear analysis. In terms of non-linear analysis of FGB, very few works have been devoted to non-linear free vibrations of cracked FGB. Kitipornchai et al. investigated the non-linear free vibration of FG cracked Timoshenko beam using the Ritz method and the rotational spring model [18]. Akbaş used the total Lagrangian finite element method to investigate the geometrically non-linear vibration of an edge cracked Timoshenko beam [19] and more recently Panigrahi and Pohit studied the free vibration of a cracked FGB, based on the neutral surface approach, the Ritz method and an iterative technique [20]. To the author's best knowledge, the researches regarding non-linear forced vibration responses of cracked FGB are very limited. Yan et al. studied the non-linear dynamic response of FG Timoshenko beam with an edge crack under a parametric excitation, combining a static compressive load and a harmonic excitation force [21]. Panigrahi and Pohit studied the non-linear dynamics of a FG cracked Timoshenko beam under an excitation force using the harmonic balance method in conjunction with an iterative technique [22]. The readers interested in more details on this topic can refer to [23]. The present article is an extension of our previous work [24], based on the single mode approach. The objective was particularly focused on the prediction of the nonlinear frequency response curves in the neighbourhood of the resonances considered. The applicability of this approach shows that the nonlinear effect does not appear only via the amplitude frequency dependence but also via the amplitude dependence of the cracked FGB deflection shapes. The purpose of the present contribution is the application of the non-linear forced vibration model previously reported in [25] using a multimode response approach and combined with the homogenization procedure based on the neutral surface approach previously adopted in [26,27]. This allowed an explicit calculation of multidimensional non-linear frequency response curves, the corresponding amplitude dependence deflection shapes and the associated curvatures distributions of a multi-cracked FGB subjected to a uniformly distributed harmonic force applied over the beam length. The closed-form solutions and the transfer matrix method used previously in [28, 29] are employed here and the resulting frequency equation is solved iteratively by the Newton Raphson algorithm. The improved model developed in [30] is used to obtain the multi-cracked beam non-linear deflection shapes and the dependence frequency amplitudes (backbone curves) at large vibration amplitudes. A parametric study is performed to explore the effects of the gradient of material properties, the vibration amplitudes, the crack parameters and the applied harmonic force on the non-linear forced dynamic behaviour of multicracked FGB.

\section{PROBLEM FORMULATION}

\subsection{Mechanical properties of the FG multi- cracked beam}

Consider the straight functionally graded EulerBernoulli uniform beam, with $N$ multi-cracks located at the positions $x_{c_{j}}$, having the geometrical characteristics shown in Fig. 1. As may be seen, the FGB, supported by linear and rotational springs at both ends, is subjected to harmonic excitation forces.

$$
\begin{aligned}
& E(z)=E_{1} \sqrt{k} e^{\frac{z}{h} \ln (k)} \\
& \rho(z)=\rho_{1} \sqrt{k} e^{\frac{z}{h} \ln (k)}
\end{aligned}
$$

with $E_{1}, \rho_{1}$ being the elastic modulus and the material density at the FGB top surface respectively. $(z=0)$ corresponds to the middle surface. It should be emphasized that the Poisson's ratio $v$ is considered constant throughout the analysis because of its weak influence on the stress intensity factor (SIF) [11]. The index $k=E_{2} / E_{1}$ is introduced as a material property ratio.

\subsection{Homogenisation procedure based on the neutral surface approach}

In the case of FGB, the material property changes in the thickness direction are not symmetric, and consequently, the neutral plane does not coincide with the middle plane. As a result, the bendingextensional coupling effect occurs, as was observed in [18]. The distance separating the two planes can be expressed as follows [22]:

$$
\delta=\int_{-\frac{h}{2}}^{\frac{h}{2}} z E(z) d z / \int_{-\frac{h}{2}}^{\frac{h}{2}} E(z) d z
$$

The formulation presented in what follows is based on the neutral surface approach. By considering the 


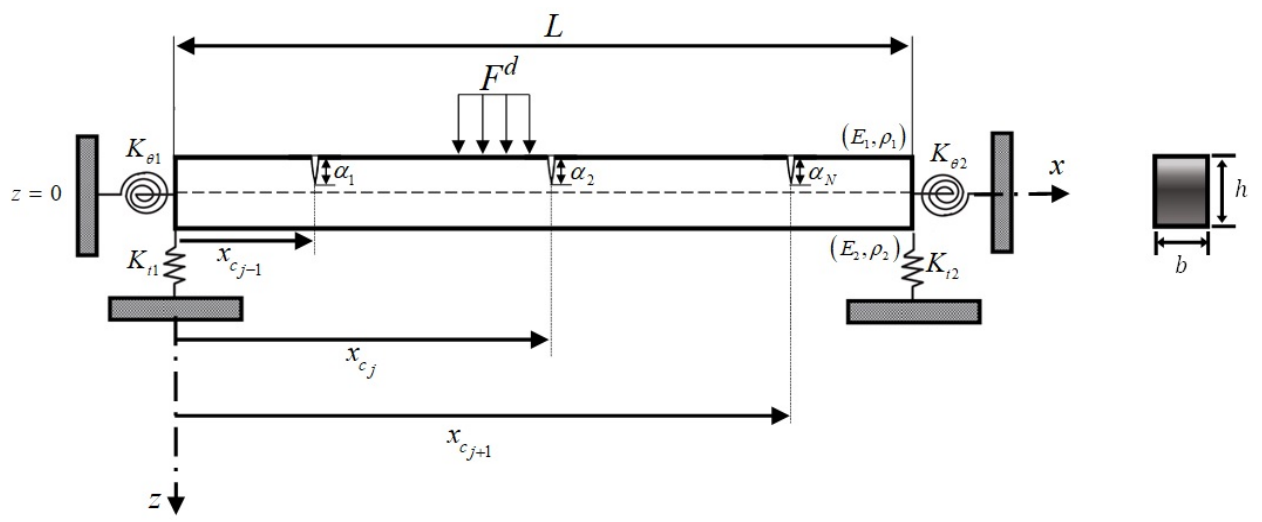

Fig. 1. Physical model of the multi-cracked FGB

change of variable $\bar{z}=z+\delta$, the coupling between the extension and bending is eliminated from the equations and the effective parameters of the equivalent homogeneous beam are defined as explained below (equations 14 to 16 and equation 32).

\subsection{Rotational spring model}

The cracked FGB is modelled as an assembly of uniform sub-segments connected by massless rotational springs as in [31] presenting the reduced local flexibility calculated using the fracture mechanics theory. It is assumed that the crack, perpendicular to the beam surface, remains always open. The bending stiffness $K_{\tau}$ of the cracked section is related to the flexibility $C$ by [26]:

$$
C=\int_{0}^{\alpha} \frac{72 \pi\left(1-v^{2}\right) \alpha f^{2}(\alpha / h, k)}{E(\alpha) h^{2}} d \alpha=\frac{1}{K_{\tau}}
$$

With $E(\alpha)$ is the effective elastic modulus at the crack tip defined as:

$$
E(\alpha)=E_{1} \sqrt{k} e^{\left(-\frac{1}{2}+\frac{\alpha-\delta}{h}\right) \ln (k)}
$$

Where $\alpha / h$ is the crack depth and $f(\alpha / h, k)$ is a crack correction function of two independent variables. Through a number of numerical experiments, the function $f$ has been expressed as a rational function [5]:

$$
f(\alpha / h, k)=\frac{N U M}{D E N}
$$

Such as:

$$
\begin{aligned}
& N U M=1.1732-0.3539 \ln (k)+0.0289[\ln (k)]^{2} \\
& -0.0061[\ln (k)]^{3}+0.6625(\alpha / h)+3.072(\alpha / h)^{2}
\end{aligned}
$$

and

$$
\begin{aligned}
& D E N=1-0.0014 \ln (k)-0.0017[\ln (k)]^{2} \\
& +1.9917(\alpha / h)-3.0982(\alpha / h)^{2}-3.0982(\alpha / h)^{3}
\end{aligned}
$$

\subsection{Linear vibration analysis}

Based on the Euler-Bernoulli beam theory, the longitudinal displacement $U$ and the transverse displacement $W$ of an arbitrary point along the $x$ and $z$ axes are obtained as:

$$
\left\{\begin{array}{c}
U(x, \bar{z}, t)=u(x, t)-\bar{z} \frac{\partial w}{\partial x} \\
W(x, \bar{z}, t)=w(x, t)
\end{array}\right.
$$

where $u$ and $w$ are the displacement of a current neutral line point. The strains are given by:

$$
\varepsilon_{x}=\frac{\partial u}{\partial x}-\bar{z} \frac{\partial^{2} w}{\partial x^{2}}
$$

The stress-strain relation given by the generalised Hooke's law is:

$$
\sigma_{x}=\frac{E(\bar{z})}{\left(1-v^{2}\right)} \varepsilon_{x}
$$

The force and bending moment resultant expressions are:

$$
\begin{gathered}
\overline{N_{x}}=\int_{A} \sigma_{x} d A=\overline{A_{11}}\left[\frac{\partial u}{\partial x}+\frac{1}{2}\left(\frac{\partial w}{\partial x}\right)^{2}\right]-\overline{B_{11}} \frac{\partial^{2} w}{\partial x^{2}} \\
\overline{M_{x}}=\int_{A} \sigma_{x} \bar{z} d A=\overline{B_{11}}\left[\frac{\partial u}{\partial x}+\frac{1}{2}\left(\frac{\partial w}{\partial x}\right)^{2}\right]-\overline{D_{11}} \frac{\partial^{2} w}{\partial x^{2}}
\end{gathered}
$$

$\overline{A_{11}}, \overline{B_{11}}$ and $\overline{D_{11}}$ are the extensional, coupling and bending rigidity coefficients defined by:

$$
\left(\overline{A_{11}}, \overline{B_{11}}, \overline{D_{11}}\right)=\int_{-\frac{h}{2}-\delta}^{\frac{h}{2}-\delta} \frac{E(\bar{z})}{\left(1-v^{2}\right)}\left(1, \bar{z}, \bar{z}^{2}\right) \mathrm{d} \bar{z}
$$

Taking into account Eq. (3) and integrating the coefficients in Eq. (14) leads to $\overline{B_{11}}=0$. Neglecting the axial inertia, the equations of motion for the $i^{\text {th }}$ sub-beam can be derived as:

$$
\begin{gathered}
\frac{\partial \overline{N_{x}}}{\partial x}=0 \\
\frac{\partial^{2} \overline{M_{x}}}{\partial x^{2}}-I_{1} \frac{\partial^{2} w}{\partial t^{2}}=(E I)_{\mathrm{eff}} \frac{\partial^{4} w}{\partial x^{4}}+(\rho S)_{\mathrm{eff}} \frac{\partial^{2} w}{\partial t^{2}}=0
\end{gathered}
$$


Equation (16) is effective for replacing the FGB by an equivalent isotropic beam with an effective bending stiffness and a mass per unit length given by: $(E I)_{\mathrm{eff}}=\left(D_{11}-B_{11}^{2} / A_{11}\right)$ and $(\rho S)_{\mathrm{eff}}=I_{1}$, with $I_{1}$ defined by:

$$
I_{1}=\int_{-\frac{h}{2}-\delta}^{\frac{h}{2}-\delta} \rho(\bar{z}) d \bar{z}
$$

Integrating Eq. (15) with respect to $x$ and using the immovable end conditions $u=0$ at $x=0$ and $x=L$ leads to:

$$
\overline{N_{x}}=\overline{A_{11}} \int_{0}^{L}\left(\frac{\partial w}{\partial x}\right)^{2} d x
$$

The above expression for $\overline{N_{x}}$ corresponds to that of an equivalent isotropic beam with $\overline{A_{11}}=(E S)_{\text {eff }}$. Assuming harmonic vibrations, the transverse displacements in the $j^{\text {th }}$ span can be written as:

$$
\begin{aligned}
& w_{i j}\left(x^{*}\right)=A_{j} \cosh \left(\beta_{i} L\left(x^{*}-\xi_{j-1}\right)\right)+B_{j} \sinh \left(\beta_{i} L\left(x^{*}-\xi_{j-1}\right)\right) \\
& +C_{j} \cos \left(\beta_{i} L\left(x^{*}-\xi_{j-1}\right)\right)+D_{j} \sin \left(\beta_{i} L\left(x^{*}-\xi_{j-1}\right)\right)
\end{aligned}
$$

$$
\xi_{j-1} \leq x^{*} \leq \xi_{j} \text { for } j=1,2, \ldots, N+1
$$

in which $x^{*}=x / L$ and $\xi_{j}=x_{c_{j}} / L$. The beam eigenvalue frequency parameter $\beta_{i}$, corresponding to the beam $i^{\text {th }}$ mode shape ( $i=1$ to $n$ ) is given by:

$$
\beta_{i}^{4}=\omega_{i}^{2} \frac{(\rho S)_{\mathrm{eff}}}{(E I)_{\mathrm{eff}}}
$$

The constants $\left(A_{j}, B_{j}, C_{j}, D_{j}\right)$ are determined by the beam end conditions:

$$
\begin{aligned}
& \left\{\left.\frac{d^{3} w_{1 i}\left(x^{*}\right)}{d x^{* 3}}\right|_{x^{*}=0}=-\left.K_{t 1} w_{1 i}\left(x^{*}\right)\right|_{x^{*}=0}\right. \\
& \left.\frac{d^{2} w_{1 i}\left(x^{*}\right)}{d x^{* 2}}\right|_{x^{*}=0}=\left.K_{\theta 1} \frac{d w_{1 i}\left(x^{*}\right)}{d x^{*}}\right|_{x^{*}=0} \\
& \left\{\left.\frac{d^{3} w_{(\mathrm{N}+1) i}\left(x^{*}\right)}{d x^{* 3}}\right|_{x^{*}=1}=\left.K_{t 2} w_{(\mathrm{N}+1) i}\left(x^{*}\right)\right|_{x^{*}=1}\right. \\
& \left.\frac{d^{2} w_{(\mathrm{N}+1) i}\left(x^{*}\right)}{d x^{* 2}}\right|_{x^{*}=1}=-\left.K_{\theta 2} \frac{d w_{(\mathrm{N}+1) i}\left(x^{*}\right)}{d x^{*}}\right|_{x^{*}=1}
\end{aligned}
$$

and the continuity and compatibility conditions at the $j^{\text {th }}$ crack location $\xi_{j}$ [32]:

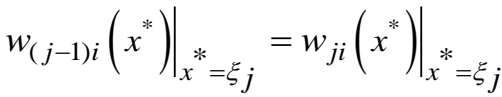

$$
\begin{aligned}
& \left.\frac{\mathrm{d}^{2} w_{(j-1) i}}{\mathrm{~d} x^{* 2}}\right|_{x^{*}=\xi_{j}}=\left.\frac{\mathrm{d}^{2} w_{j i}}{\mathrm{~d} x^{* 2}}\right|_{x=\xi_{j}} \\
& \left.\frac{\mathrm{d}^{3} w_{(j-1) i}}{\mathrm{~d} x^{* 3}}\right|_{x^{*}=\xi_{j}}=\left.\frac{\mathrm{d}^{3} w_{j i}}{\mathrm{~d} x^{* 3}}\right|_{x=\xi_{j}}
\end{aligned}
$$

$$
\left.\frac{\mathrm{d} w_{(j-1) i}}{\mathrm{~d} x^{*}}\right|_{x=\xi_{j}}=\left.\frac{\mathrm{d} w_{j i}}{\mathrm{~d} x^{*}}\right|_{x=\xi_{j}}+\left.\frac{L}{K^{*}} \frac{\mathrm{d}^{2} w_{j i}}{\mathrm{~d} x^{* 2}}\right|_{x^{*}=\xi_{j}}
$$

where $K_{t 1}, K_{t 2}, K_{\theta 1}$ and s are the stiffness of the transverse and rotational springs respectively. $K^{*}$ is the non-dimensional local rigidity due to the crack, related to the local flexibility coefficient $C$ of the rotational spring by :

$$
K^{*}=\frac{L}{C E I}=\frac{K_{\tau} L}{E I}
$$

Based on the matrix transfer method as well explained previously in [29], the specified conditions lead to a homogeneous linear system, which has a non-trivial solution if its determinant is set equal to zero. The resulting equation, solved iteratively using the Newton Raphson algorithm, leads to the beam natural frequencies. The corresponding mode shapes are then calculated by the usual algebraic procedure.

\subsection{Non-linear vibration analysis}

The Von Kàrmàn type non-linear straindisplacement relationships are:

$$
\varepsilon_{x}=\frac{\partial u}{\partial x}+\frac{1}{2}\left(\frac{\partial w}{\partial x}\right)^{2}-\bar{z} \frac{\partial^{2} w}{\partial x^{2}}
$$

The elastic strain energy $V$ of the beam is given as:

$$
V=\frac{1}{2} \int_{0}^{L}\left[\overline{N_{x}} \varepsilon_{x}-\overline{M_{x}} \frac{\partial^{2} w}{\partial x^{2}}\right] \mathrm{d} x
$$

With Eq. (3) always in mind, the strain energy $V$ can be written now only in terms of the transverse displacement $w$ as follows:

$$
V=\frac{(E S)_{\mathrm{eff}}}{8 L}\left(\int_{0}^{L}\left(\frac{\partial w}{\partial x}\right)^{2}\right)^{2}+\frac{(E I)_{\mathrm{eff}}}{2} \int_{0}^{L}\left(\frac{\partial^{2} w}{\partial x^{2}}\right)^{2} \mathrm{~d} x
$$

The kinetic energy $T$ and the strain energy of the crack $V_{c}$ as mentioned in [32] are given by:

$$
\begin{gathered}
T=\frac{1}{2} \int_{0}^{L} \int_{-\frac{h}{2}-\delta}^{\frac{h}{2}-\delta} \rho(\bar{z})\left(\frac{\partial w}{\partial t}\right)^{2} \mathrm{~d} x d \bar{z} \\
V_{\mathrm{c}}=\frac{(E I)_{\mathrm{eff}}^{2}}{2 K_{\tau}}\left(\frac{\partial^{2} w}{\partial x^{2}}\right)_{x=x_{c_{N}}}
\end{gathered}
$$

The potential energy associated to the force $V_{f}$ is given by [33]:

$$
V_{f}=\int_{S} F(x, t) w_{i}(x) d x
$$

The harmonic force is uniformly distributed and equal to $F^{d}$ respectively, excites the beam $i^{\text {th }}$ mode by: 


$$
F_{i}^{d}(t)=F^{d} \sin (\omega t) \int_{0}^{L} w_{i}(x) d x=f_{i}^{d} \sin (\omega t)
$$

Considering non-linear vibrations and assuming a harmonic motion, the transverse displacement is expressed as:

$$
w(x, t)=a_{i} w_{i}(x) \sin (\omega t)
$$

in which the $a_{i}{ }^{\prime} s$ are the unknown basic function contribution coefficients. Inserting Eq. (37) into Eqs. (32-35) and applying Hamilton's principle gives:

$$
\partial \int_{0}^{\frac{2 \pi}{\omega}}\left(V+V_{c}+V_{f}-T\right) d t=0
$$

and introducing the following dimensionless parameters:

$$
w(x)=h w^{*}\left(\frac{x}{L}\right)=h w^{*}\left(x^{*}\right)
$$

A set of non-linear algebraic equations is then obtained:

$2 a_{i} K_{i r}^{*}+3 a_{i} a_{j} a_{k} B_{i j k r}^{*}-2 \omega^{* 2} a_{i} M_{i r}^{*}=F_{i}^{*} \quad r=1, \ldots, n$

Where $K_{i j}^{*}, B_{i j k l}^{*}$ and $M_{i j}^{*}$ stand for the dimensionless classical rigidity tensor, the nonlinear rigidity tensor and the mass tensor, defined as:

$$
K_{i j}^{*}=\int_{0}^{1} \frac{\partial^{2} w_{i}^{*}}{\partial x^{* 2}} \frac{\partial^{2} w_{j}^{*}}{\partial x^{* 2}} \mathrm{~d} x^{*}+\left.\left.\sum_{c=1}^{N} \frac{(E I)_{e f f}}{K_{\tau}^{c}} \frac{\partial^{2} w_{i}^{*}}{\partial x^{* 2}}\right|_{x=\xi} x^{*} \frac{\partial^{2} w_{j}^{*}}{\partial x^{* 2}}\right|_{x=\xi} ^{*}
$$

$$
B_{i j k l}^{*}=\frac{R^{2}(E S)_{\mathrm{eff}}}{4(E I)_{\mathrm{eff}}}\left(\int_{0}^{1} \frac{\partial w_{i}^{*}}{\partial x^{*}} \frac{\partial w_{j}^{*}}{\partial x^{*}} \mathrm{~d} x^{*}\right)\left(\int_{0}^{1} \frac{\partial w_{k}^{*}}{\partial x^{*}} \frac{\partial w_{l}^{*}}{\partial x^{*}} \mathrm{~d} x^{*}\right)
$$

$$
M_{i j}^{*}=\int_{0}^{1} w_{i}^{*} w_{j}^{*} d x^{*}
$$

The dimensionless generalized force $F_{i}^{d^{*}}$ is given by :

$$
F_{i}^{d^{*}}=F^{d} \frac{L^{4}}{R(E I)_{\mathrm{eff}}} \int_{0}^{1} w_{i}^{*}\left(x^{*}\right) d x^{*}
$$

Equation (40) may be written in a matrix form as:

$$
\left[K^{*}\right]\{A\}+\frac{3}{2}\left[B^{*}(\{A\})\right]\{A\}-\omega^{* 2}\left[M^{*}\right]\{A\}=\left\{F^{*}\right\}
$$

\subsection{The multi-mode approach}

The last system Eq. (45) is similar to that obtained in [33]. It can be solved by the so-called second formulation [25], in order to obtain the beam non-linear forced vibration deflection shapes and frequency response curves at large vibration amplitudes. The basic idea behind this method consists on writing the contribution vector to the non-linear mode considered as $\{A\}^{T}=\left[a_{1}, \varepsilon_{3}, \ldots, \varepsilon_{9}\right]$, in which $\varepsilon_{2}$ to $\varepsilon_{n}$ representing the basic function with a small contribution compared to $a_{1}$ that is the predominant basic functions contribution. Considering the expression $a_{i} a_{j} a_{k} B_{i j k r}^{*}$ of Eq. (40), the third and second order terms with respect to $\varepsilon_{i}$, i.e. terms of type $\varepsilon_{i} \varepsilon_{j} \varepsilon_{k} B_{i j k r}^{*}$ or of the type $\varepsilon_{i} \varepsilon_{j} a_{1} B_{i j 1 r}^{*}$ are neglected, this leads to :

$$
a_{i} a_{j} a_{k} B_{i j k r}^{*}=a_{1}^{3} B_{111 r}^{*}+a_{1}^{2} \varepsilon_{i} B_{11 i r}^{*}
$$

Substituting and rearranging permits one to write Eq. (40) in a matrix form as:

$$
\left(\left[K^{*}\right]-\omega^{* 2}\left[M^{*}\right]\right)\{A\}+\frac{3}{2}\left[\phi^{*}\right]\{A\}=\left\{F_{r}^{*}-\frac{3}{2} a_{r}^{3} B_{i r r r}^{*}\right\}
$$

In which $\left[\phi_{I}^{*}\right]$ is $9 \times 9$ square matrix, whose general term $\phi_{i j}$ equal to $a_{1}^{2} B_{i j 11}^{*}$, depends only on $a_{1}$ which can be obtained as demonstrated before in [24,34] from the single-mode approach application.

\section{NUMERICAL RESULTS AND DISCUSSION}

The numerical calculations of the present formulation have been made with a Matlab code. Through this program, it became possible to analyse the effects of the crack parameters such as the number, the location and the depth of the crack upon the vibration characteristics of cracked FGB. To test the validity and accuracy of the present analysis, following the approximated explicit method mentioned above in the case where the force is zero, the non-linear free vibration analysis is carried out and the numerical convergence is firstly performed. The first non-linear frequency ratios of a clampedclamped FGB with an edge crack computed by the classical Euler-Bernoulli beam theory are listed in Table 1 and compared to those obtained by

Table 1: Comparison of the first non-linear frequency ratio $\omega_{n l}^{*} / \omega_{l}^{*}$ of a FGB with an edge crack

\begin{tabular}{cccccccccc}
\hline$w_{\max }$ & \multicolumn{3}{c}{$k=0.2$} & & & $k=1$ & & \multicolumn{3}{c}{$k=5$} \\
\hline & Present & {$[18]$} & R. D \% & Present & {$[18]$} & R. D \% & Present & {$[18]$} & R. D \% \\
\cline { 2 - 10 } 0.2 & 1.0146 & 1.0204 & 0.57 & 1.0120 & 1.0184 & 0.63 & 1.0133 & 1.0195 & 0.61 \\
0.4 & 1.0569 & 1.0800 & 2.14 & 1.0472 & 1.0713 & 2.25 & 1.0521 & 1.0755 & 2.18 \\
0.6 & 1.1247 & 1.1724 & 4.07 & 1.1039 & 1.1529 & 4.25 & 1.1145 & 1.1615 & 4.05 \\
\hline
\end{tabular}

R. D = |Present $-[18] /[18] \mid$ 
Timoshenko beam theory developed in [18] for various vibrations amplitudes corresponding to $\alpha / h=0.2$ and $\xi_{j}=0.5$. The following material and physical parameters are adopted: the top surface of the FGM $E_{1}=70 \mathrm{Gpa}, \rho_{1}=2780 \mathrm{Kg} / \mathrm{m}^{3}$ and slenderness ratio $L / h=10$ with $h=0.1 \mathrm{~m}$. It is observed that the trend of the present results matched appropriately with that obtained in [18]. It should be noted that the FGB under investigation in the rest of the analysis, has slenderness ratio $L / h=20 \%$ with $h=0.05 \mathrm{~m}$ and that all the analysis will focus on the clamped ends obtained by taking $K_{t 1}=K_{t 2}=K_{\theta 1}=K_{\theta 2}=\infty$.

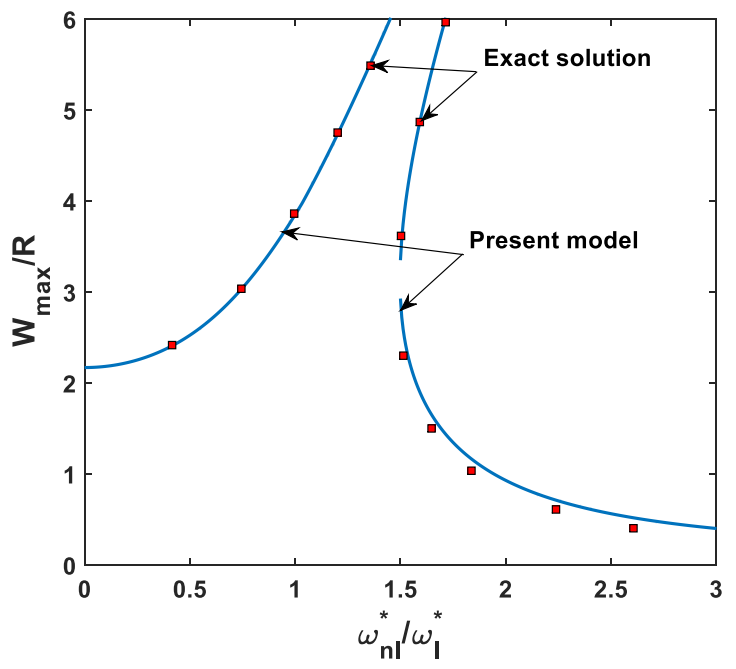

Fig. 2. Comparison between the non-linear frequency response curves of a C-C beam, based on the multi-mode approach obtained by present model and the exact solution

In order to validate the proposed procedure in the case of non-linear forced vibrations, the non-linear response curves, obtained by the present model in the neighbourhood of the first non-linear mode shape, is compared in Figure 2 with that obtained previously by the exact solution in [33], for the case of $\mathrm{C}-\mathrm{C}$ isotropic beam (in the present work $k=1$ ) excited by a harmonic concentrated force $F^{c}=500$ applied at its middle with $R=\sqrt{I / S}$ (radius of gyration). It can be concluded that a supreme agreement can be observed.

Before investigating the non-linear forced response of the cracked FGB, as described in the general formulation, it is necessary to determine the predominant mode excited in the case of a given distributed harmonic physical force, in order to justify the use of the multimode approach in its vicinity. Therefore, the percentages of the generalised distributed forces corresponding to the first five symmetrical modes, in the case of a C-C FGB with an edge crack at its center, applied over the whole beam span, corresponding to $k=5$ are listed in Table 2. It can evidently be concluded that the force predominantly excites the first mode.

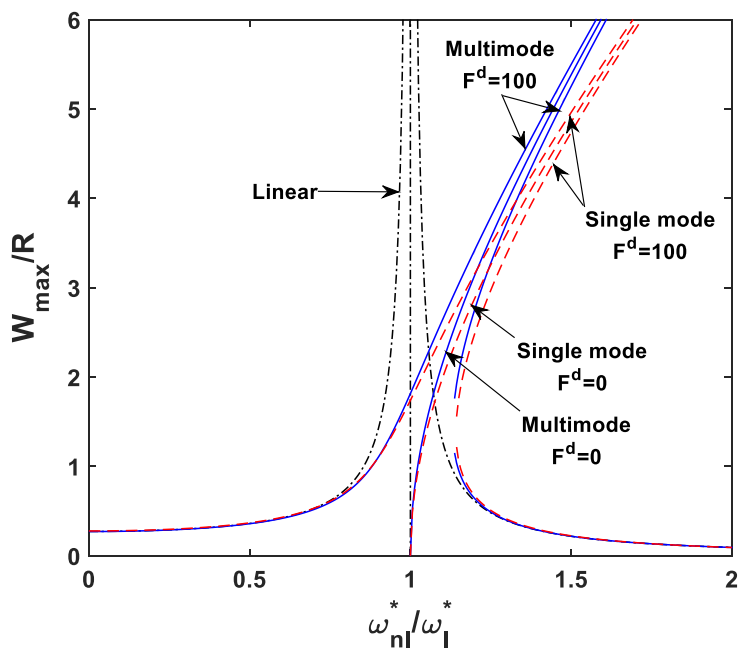

Fig. 3. Comparison with the linear and nonlinear frequency response curves obtained by the single mode approach and the multimode approach of a C-C FGB with three edge cracks

In Figure 3, a comparison is made between the linear and the non-linear frequency response curves obtained by the single-mode approach and the multimode approach of a C-C FGB with three edge cracks equitably distributed along the beam length, in the case when the FGB is excited by a harmonic distributed force $F^{d}=100$ along the whole cracked FGB, corresponding to $\alpha_{(1,2,3)} / h=0.1$. As can be seen, considering the effect of geometric nonlinearity, the frequency response curve is increased and tends to the right showing the hardening behaviour. Furthermore, an important conclusion that can be deduced is that the two curves obtained in the non-linear analysis are very close to each other for maximum vibration amplitudes $w_{\max } / R$ up to 1.5

Table 2. Percentages of generalised distributed forces exciting the first five symmetric modes of a C-C FGB with an edge crack at its center.

\begin{tabular}{cccccc}
\hline Modes & 1 & 3 & 5 & 7 & 9 \\
\hline $\int_{0}^{1} w_{i}^{*}(x)$ & $8.2939 \mathrm{E}-01$ & $3.6535 \mathrm{E}-01$ & $2.3237 \mathrm{E}-01$ & $1.7041 \mathrm{E}-01$ & $1.3454 \mathrm{E}-01$ \\
\hline $\int_{0}^{1} w_{i}^{*}(x) / \sum_{i=1}^{n}\left|\int_{0}^{1} w_{i}^{*}(x)\right|$ & 48 & 21 & 13 & 10 & 8
\end{tabular}




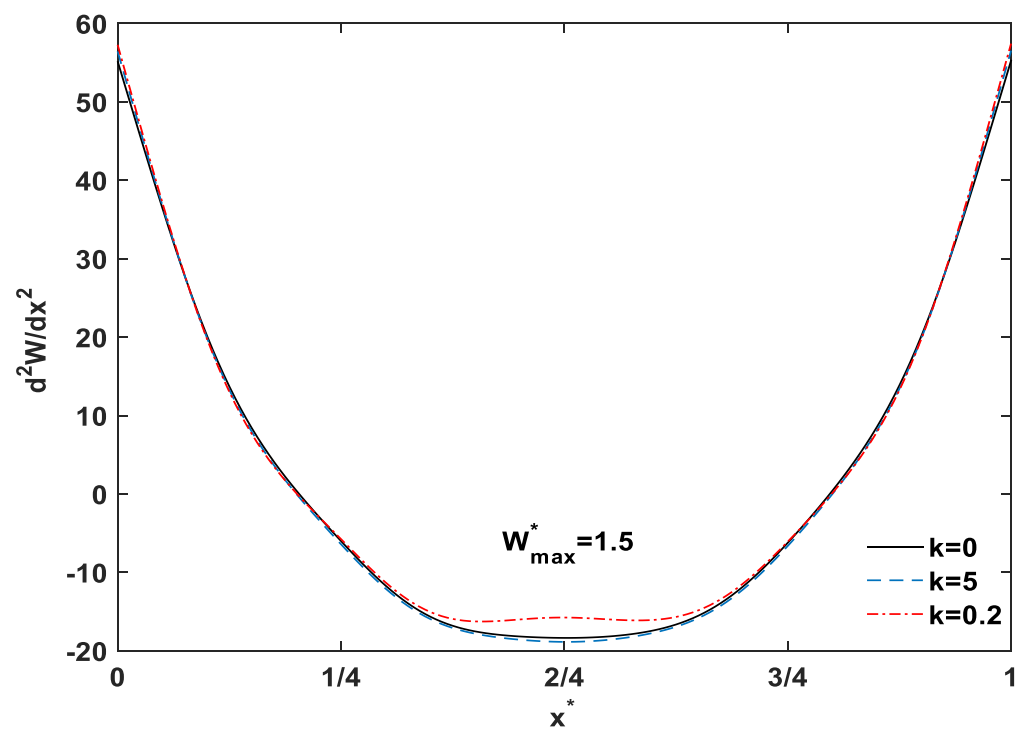

Fig. 4. The curvature distributions associated to the first nonlinear deflection response function of a C-C FGB with three cracks corresponding to various values of the materiel properties

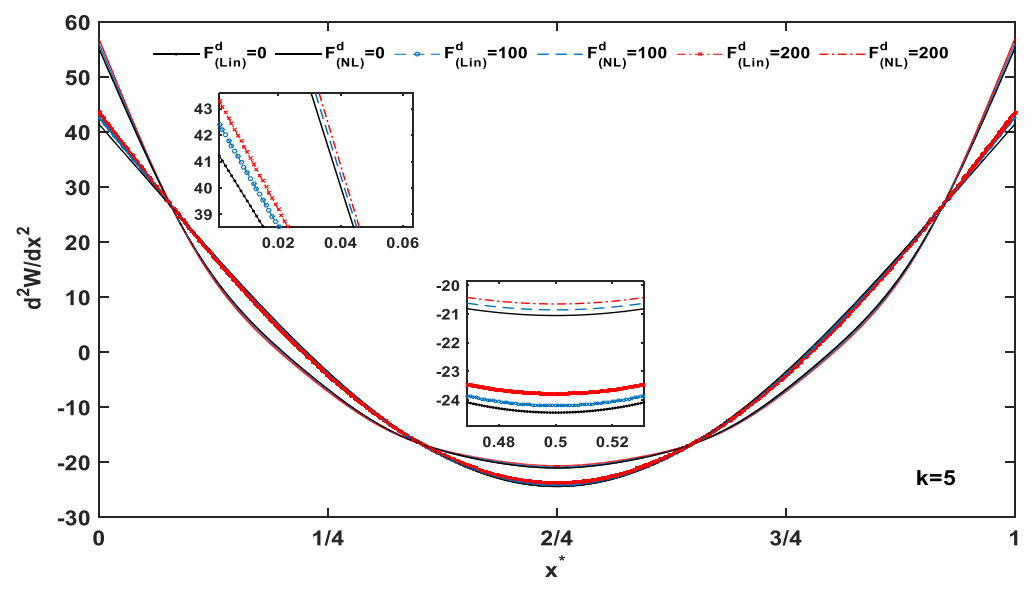

Fig. 5. The curvature distributions associated to the first nonlinear deflection response function of a C-C FGB having $k=5$ with an edge crack corresponding to various values of the harmonic distributed force

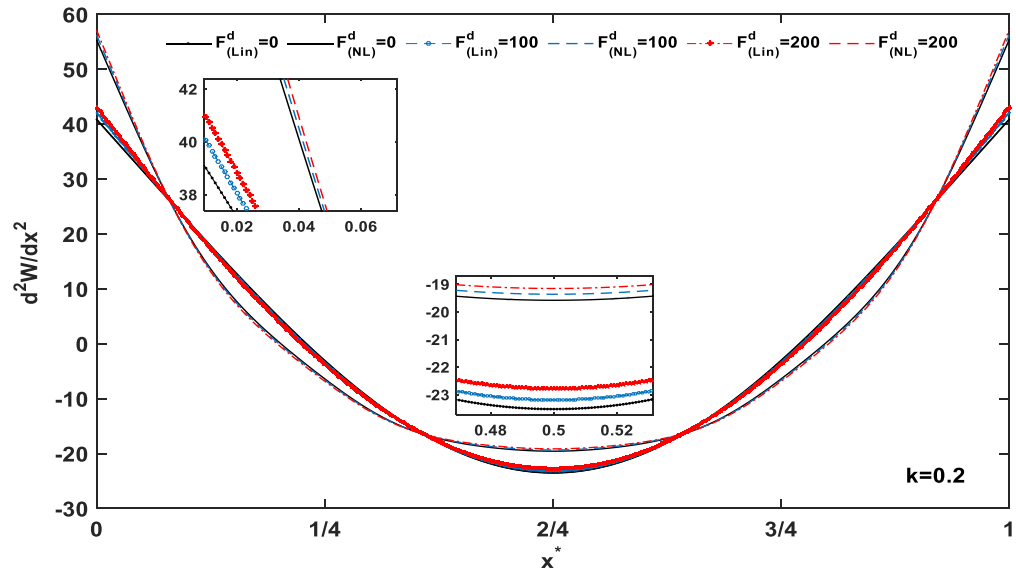

Fig. 6. The curvature distributions associated to the first nonlinear deflection response function of a C-C FGB having $k=0.2$ with an edge crack corresponding to various values of the harmonic distributed force

and slight differences start to appear for higher vibration amplitudes. This fact provides a clear explanation for the small influence of the contributions of the higher functions on the frequency response, compared to those obtained by the single-mode approach. 
Table 3. Effect of force variation on the percentage correction introduced by the non-linear theory compared to the linear theory

\begin{tabular}{|c|c|c|c|c|c|c|c|c|}
\hline & & & \multicolumn{2}{|c|}{$d^{2} w(0) / d x^{2}$} & \multirow{2}{*}{$\%$} & \multicolumn{2}{|c|}{$d^{2} w(1 / 2) / d x^{2}$} & \multirow{2}{*}{$\%$} \\
\hline & & & Lin & $\mathrm{NL}$ & & Lin & NL & \\
\hline \multirow{6}{*}{$w_{\max }=1.5$} & \multirow{3}{*}{$k=5$} & $F^{d}=0$ & 41.41 & 55.19 & 24.97 & 24.43 & 21.05 & 16.06 \\
\hline & & $F^{d}=100$ & 42.61 & 56.08 & 24.02 & 24.19 & 20.85 & 16.02 \\
\hline & & $F^{d}=200$ & 43.51 & 56.91 & 23.55 & 23.78 & 20.64 & 15.21 \\
\hline & \multirow{3}{*}{$k=0.2$} & $F^{d}=0$ & 40.66 & 55.36 & 26.55 & 23.39 & 19.57 & 20.13 \\
\hline & & $F^{d}=100$ & 42.06 & 56.23 & 25.20 & 23.18 & 19.36 & 19.73 \\
\hline & & $F^{d}=200$ & 43.1 & 57.13 & 24.56 & 22.78 & 19.15 & 18.96 \\
\hline
\end{tabular}
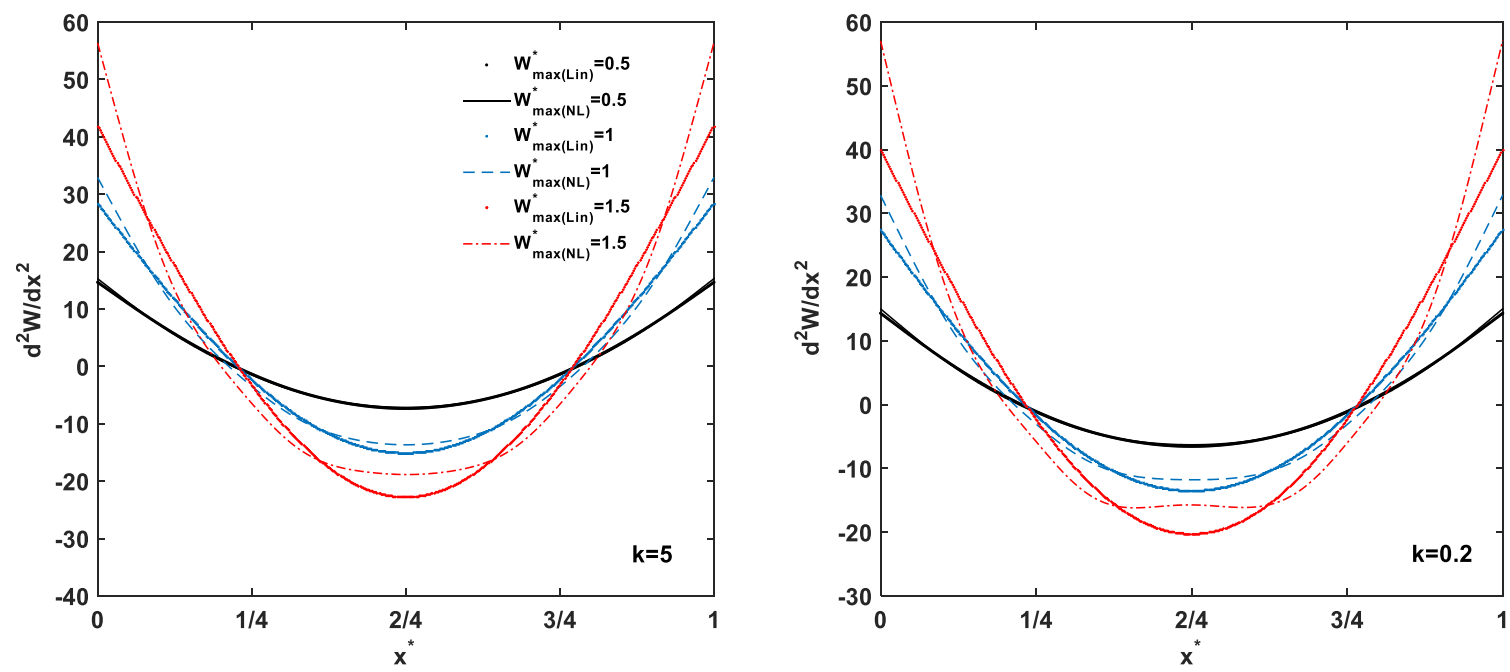

Fig. 7. The curvature distributions associated to the first nonlinear deflection response function of a C-C FGB with three cracks corresponding to various values of the vibration amplitudes

The effect of a uniformly harmonic distributed force $F^{d}=100$ applied along the whole of the FGB with three cracks equitably distributed along the beam length corresponding to $\alpha_{(1,2,3)} / h=0.3$ and a maximum non-dimensional amplitudes up to more than once the beam thickness $w_{\max } / R=1.5$, for various values of materiel properties is depicted in Figure 4. It can be clearly observed that the nonlinear curvature distribution curve in the case of the FGB having material property $k=0.2$ i.e. "top surface rich ceramic" is hardly affected and more showed exactly where the crack is located (at the beam center), compared to the FGB with $k=5$ i.e. "top surface rich metal".

The associated curvature distributions to the first nonlinear deflection obtained via the present model for a C-C FGB containing three edge cracks distributed along the beam length and excited by various values of a uniformly harmonic distributed force applied along the whole of the beam for both scenarios, when the FGB having material property $k=5$ and $k=0.2$ respectively are plotted in Figure 5 and 6 , for maximum non-dimensional amplitudes $w_{\max } / R=1.5$, corresponding to $\alpha_{(1,2,3)} / h=0.2$. As can be seen, increasing the excitation level leads to

Table 4. Effect of vibration amplitude on the percentage correction introduced by the non-linear theory compared to the

\begin{tabular}{|c|c|c|c|c|c|c|c|c|}
\hline & & & \multicolumn{2}{|c|}{$d^{2} w(0) / d x^{2}$} & \multirow{2}{*}{$\%$} & \multicolumn{2}{|c|}{$d^{2} w(1 / 2) / d x^{2}$} & \multirow{2}{*}{$\%$} \\
\hline & & & Lin & NL & & Lin & NL & \\
\hline \multirow{6}{*}{$F^{d}=100$} & \multirow{3}{*}{$k=5$} & $w_{\max }=0.5$ & 14.66 & 15.28 & 4.06 & 7.33 & 7.15 & 2.52 \\
\hline & & $w_{\max }=1$ & 28.36 & 32.92 & 13.85 & 15.14 & 13.67 & 10.75 \\
\hline & & $w_{\max }=1.5$ & 41.83 & 56.37 & 25.79 & 22.81 & 18.85 & 21.01 \\
\hline & \multirow{3}{*}{$k=0.2$} & $w_{\max }=0.5$ & 14.19 & 14.86 & 4.51 & 6.543 & 6.294 & 3.96 \\
\hline & & $w_{\max }=1$ & 27.36 & 32.83 & 16.66 & 13.55 & 11.79 & 14.93 \\
\hline & & $w_{\max }=1.5$ & 39.93 & 57.06 & 30.02 & 20.33 & 15.73 & 29.24 \\
\hline
\end{tabular}



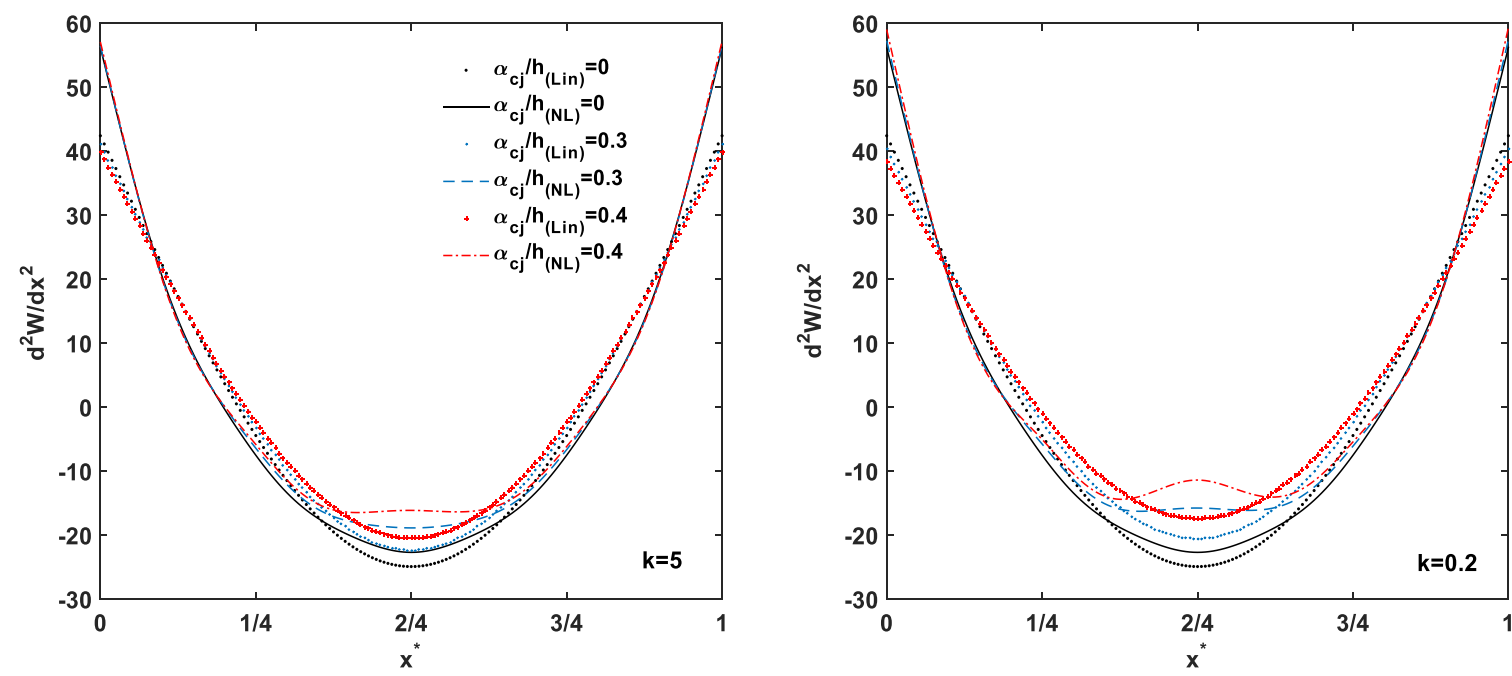

Fig. 8. The curvature distributions associated to the first nonlinear deflection response function of a C-C FGB with three cracks corresponding to various values of the crack depth

Table 5. Effect of crack depth on the percentage correction introduced by the non-linear theory compared to the

\begin{tabular}{|c|c|c|c|c|c|c|c|c|}
\hline & & & \multicolumn{2}{|c|}{$d^{2} w(0) / d x^{2}$} & \multirow{2}{*}{$\%$} & \multicolumn{2}{|c|}{$d^{2} w(1 / 2) / d x^{2}$} & \multirow{2}{*}{$\%$} \\
\hline & & & Lin & NL & & Lin & NL & \\
\hline \multirow{6}{*}{$F^{d}=100$} & \multirow{3}{*}{$k=5$} & $\alpha_{c_{j}} / h=0$ & 42.43 & 56.22 & 24.53 & 24.91 & 22.69 & 9.78 \\
\hline & & $\alpha_{c_{j}} / h=0.3$ & 41.02 & 56.45 & 27.33 & 22.35 & 18.87 & 18.44 \\
\hline & & $\alpha_{c_{j}} / h=0.4$ & 39.87 & 57.09 & 30.16 & 20.46 & 16.13 & 26.84 \\
\hline & \multirow{3}{*}{$k=0.2$} & $\alpha_{c_{j}} / h=0$ & 42.43 & 56.22 & 24.53 & 24.91 & 22.69 & 9.78 \\
\hline & & $\alpha_{c_{j}} / h=0.3$ & 40.32 & 57.28 & 29.61 & 20.53 & 15.76 & 30.27 \\
\hline & & $\alpha_{c_{j}} / h=0.4$ & 38.32 & 59.03 & 35.08 & 17.42 & 11.38 & 53.08 \\
\hline
\end{tabular}

an increase in the stress near to the clamps, on the contrary a decrease in the middle of the cracked FGB.

Our simulation brings also an important results listed in Table 3, about the effect of the distributed force variation on the percentage of correction introduced by taking into account the geometric nonlinearity effect in the analysis compared to the linear case. It has been found that when the intensity of the applied force increases, the percentage of correction decreases at both, near to the clamps and the middle of the cracked FGB and remains always higher in the case of a FGB with $k=0.2$ compared to a FGB with $k=5$.

The effect of the vibration amplitudes on the nonlinear curvature distribution associated to the nonlinear deflection shapes, of a C-C FGB with three cracks equitably distributed along the beam length subjected to a uniformly harmonic distributed force $F^{d}=100$ corresponding to $\alpha_{(1,2,3)} / h=0.3$ in the two scenarios of the FGB is plotted in Fig. 7. This figure clearly shows the influence of the nonlinear effect as expected, i.e. the increase in the stress distribution in accordance with the increase of the vibration amplitude, especially at the middle of the beam and in the regions of the clamps. It is also noticeable that the shape of the curvature changes and more specifically in the middle where the crack is located for the case of a FGB with $k=0.2$ compared to that of a FGB with $k=5$, which implies that the FGB becomes slightly flexible when the crack starts to propagate in the totally surface rich ceramic. As can be seen from Table 4, the percentage of correction introduced by the non-linear theory compared to the linear theory increases by increasing the vibration amplitude either in the middle of the beam or in the region of the clamps, and also shows us that there is no proportionality on the stress variation with the amplitude variation contrary to the linear case because the principle of supposition applied in this domain remains invalid and remains completely fails to predict the correct behaviour of the beam response at large vibration amplitudes.

Figure 8 shows the effect of crack depth on the non-linear curvature distributions of a C-C FGB with three cracks equitably distributed along the FGB length excited by a uniformly harmonic distributed force $F^{d}=100$ vibrating with significantly large amplitude $w_{\max } / R=1.5$. It is well noticed that the evolution of the crack depth is rather important and 

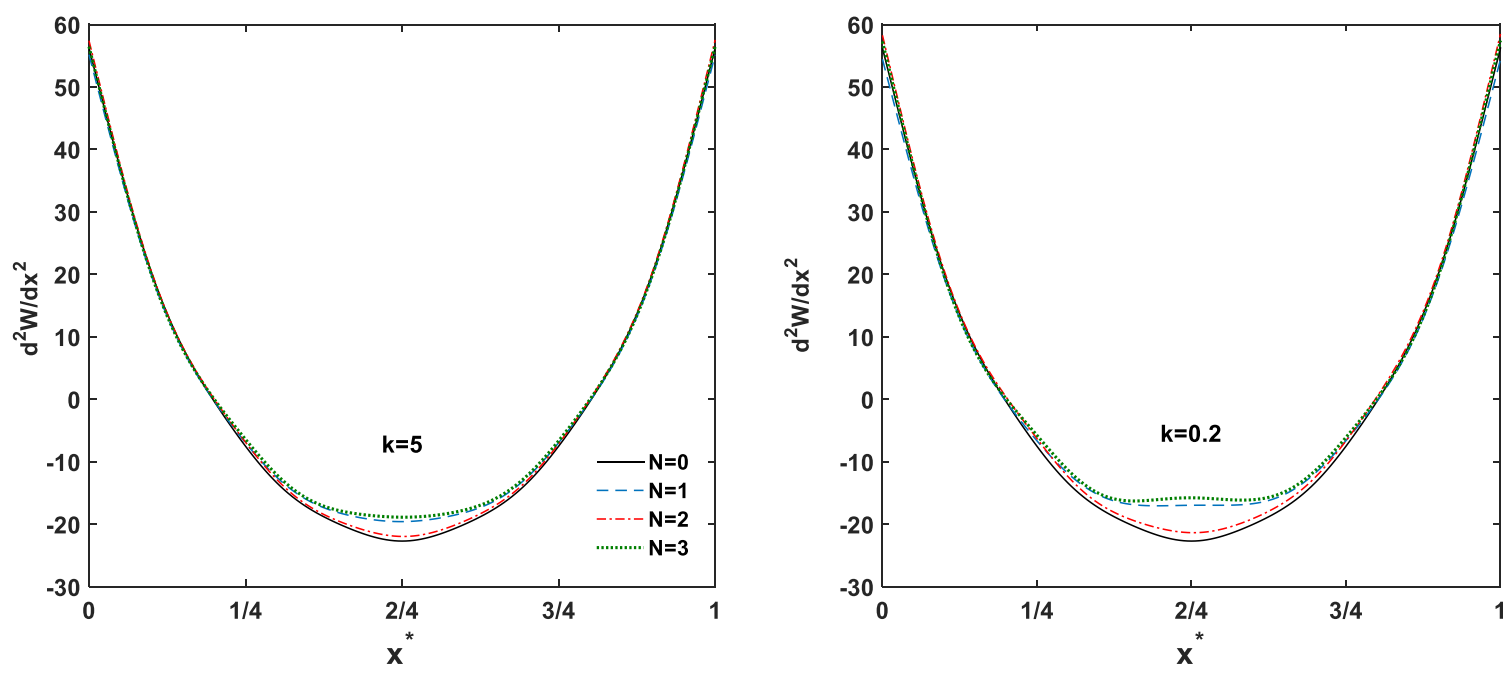

Fig. 9. The curvature distributions associated to the first nonlinear deflection response function of a C-C cracked FGB corresponding to various crack number

became more pronounced in the case of a FGB with $k=0.2$ compared to that of a FGB with $k=5$, the curvature decreases at the crack location when the crack depth increases, this clearly states that this phenomenon "softening" is caused by the flexibility of the beam. On the other hand, an inverted variation of curvature is obtained here at the regions of the clamps in which the curvature increases with increasing depth in the non-linear case and decreases in the linear case. It can also be deduced from the Table 5 that the crack depth effect increases the percentage correction of the curvature variation at the clamps regions and decreases it in the middle of the beam where the crack is located. The effect of a uniformly harmonic distributed force

A peculiar phenomenon can be also observed in Fig. 9, showing the effect of the crack number equitably distributed along the beam length with value of crack depth $\alpha / h=0.3$ on the non-linear curvature distributions corresponding to a FGB excited by a uniformly distributed force $F^{d}=100$. The variation of crack number is obviously noticed especially for the beam having material index $k=0.2$ compared to FGB with $k=5$, and it can be concluded that the crack has more effect when it is located in the middle comparing when it is located at the points considered as nodes.

\section{CONCLUSION}

The semi-analytical approach to the non-linear dynamic response of beams based on a multi-mode analysis previously developed by Benamar and coworkers has been applied in the present study to the non-linear forced vibration of functionally graded beams with multi-cracks subjected to a harmonic force for the clamped boundary conditions case. A homogenization procedure has been proposed based on the neutral surface approach leading to a simplified formulation of the non-linear problem which is reduced to that of an equivalent isotropic homogeneous multi-cracked beam without altering the accuracy of the method of analysis. A numerical solution using a simplified approximate method (the so-called second formulation) enabled the non-linear multimode frequency response function to be obtained as a solution of the multi-dimensional Duffing equation. This permitted later to obtain the non-linear forced deflection shapes and associated curvatures of multi-cracked FGB.

A comparison of the response curve of an intact isotropic beam subjected to a concentrated force with that obtained by an exact solution is carried out in order to test the validity of the proposed method. An excellent agreement had been observed.

In addition, the accuracy and convergence of the present model and of the crack modelling using the classical Euler-Bernoulli beam theory are validated by comparison with the literature results obtained by the Timoshenko beam theory in the case of a fully clamped FGB having an edge crack. An appropriate correspondence has been obtained in the tendency of the first non-linear frequency ratios according to the increase of the crack depth.

A parametric study and numerical results are also given in the form of bending moment distributions or curvatures allowing the analysis of numerous effects such as the material property, the presence of the crack and the applied harmonic distributed force on the non-linear dynamic response which can be useful in the prediction of the fatigue life of a functionally graded beam containing multi-cracks and subjected to large vibration amplitudes, providing a quantitative estimate of the percentage correction introduced by the non-linear theory compared to the linear theory.

\section{REFERENCES}

1. Sridhar R, Chakraborty A, Gopalakrishnan S. Wave propagation analysis in anisotropic and inhomogeneous uncracked and cracked structures using pseudospectral finite element method. 
International Journal of Solids and Structures. 2006; 43(16):4997-5031.

https://doi.org/10.1016/j.ijsolstr.2005.10.005.

2. Yang J, Chen Y. Free vibration and buckling analyses of functionally graded beams with edge cracks. Composite Structures. 2008; 83(1):48-60.

3. Ke L-L, Yang J, Kitipornchai S, Xiang Y. Flexural vibration and elastic buckling of a cracked timoshenko beam made of functionally graded materials. Mechanics of Advanced Materials and Structures. 2009;16(6):488-502. https://doi.org/10.1080/15376490902781175.

4. Matbuly MS, Ragb O, Nassar M. Natural frequencies of a functionally graded cracked beam using the differential quadrature method. Applied Mathematics and Computation. 2009; 215(6):2307-2316. https://doi.org/10.1016/j.amc.2009.08.026.

5. Yu Z, Chu F. Identification of crack in functionally graded material beams using the p-version of finite element method. Journal of Sound and Vibration. 2009;325(1):69-84

https://doi.org/10.1016/j.jsv.2009.03.010.

6. Ferezqi HZ, Tahani M, Toussi HE. Analytical approach to free vibrations of cracked Timoshenko beams made of functionally graded materials. Mechanics of Advanced Materials and Structures. 2010;17(5):353-365. https://doi.org/10.1080/15376494.2010.488608.

7. Van Lien T, Duc NT, Khiem NT. A new form of frequency equation for functionally graded Timoshenko beams with arbitrary number of open transverse cracks. Iranian Journal of Science and Technology, Transactions of Mechanical Engineering. 2019; 43(1):235-250. https://doi.org/10.1007/s40997-018-0152-2.

8. Shabani S, Cunedioglu Y. Free vibration analysis of functionally graded beams with cracks. Journal of Applied and Computational Mechanics. 2019. https://doi.org/10.22055/jacm.2019.30065.1672.

9. Kou KP, Yang Y. A meshfree boundary-domain integral equation method for free vibration analysis of the functionally graded beams with open edged cracks. Composites Part B: Engineering. 2019; 156:303-309.

https://doi.org/10.1016/j.compositesb.2018.08.089.

10. Birman V, Byrd LW. Vibrations of damaged cantilever beams manufactured from functionally graded materials. AIAA Journal. 2007; 45(11):27472757. https://doi.org/10.2514/1.30076.

11. Wei D, Liu Y, Xiang Z. An analytical method for free vibration analysis of functionally graded beams with edge cracks. Journal of Sound and Vibration. 2012; 331(7):1686-1700. https://doi.org/10.1016/j.jsv.2011.11.020.

12. Yan T, Kitipornchai S, Yang J, He XQ. Dynamic behaviour of edge-cracked shear deformable functionally graded beams on an elastic foundation under a moving load. Composite Structures. 2011; 93(11):2992-3001. https://doi.org/10.1016/j.compstruct.2011.05.003.

13. Yang J, Chen Y, Xiang Y, Jia XL. Free and forced vibration of cracked inhomogeneous beams under an axial force and a moving load. Journal of Sound and Vibration. 2008;312(1):166-181. https://doi.org/10.1016/i.jsv.2007.10.034.

14. Yan T, Yang J. Forced vibration of edge-cracked functionally graded beams due to a transverse moving load. Procedia Engineering. 2011; 14:3293-3300. https://doi.org/10.1016/j.proeng.2011.07.416.

15. Yan T, Kitipornchai S, Yang J. Parametric instability of functionally graded beams with an open edge crack under axial pulsating excitation. Composite Structures. 2011;93(7):1801-1808. https://doi.org/10.1016/j.compstruct.2011.01.019.

16. Lien TV, Đuc NT, Khiem NT, Lien TV, Đuc NT, Khiem NT. Free and forced vibration analysis of multiple cracked FGM multi span continuous beams using dynamic stiffness method. Latin American Journal of Solids and Structures. 2019; 16(2). https://doi.org/10.1590/1679-78255242

17. Zhu L-F, Ke L-L, Xiang Y, Zhu X-Q, Wang Y-S. Vibrational power flow analysis of cracked functionally graded beams. Thin-Walled Structures. 2020;150:106626. https://doi.org/10.1016/j.tws.2020.106626.

18. Kitipornchai S, Ke LL, Yang J, Xiang Y. Nonlinear vibration of edge cracked functionally graded Timoshenko beams. Journal of Sound and Vibration. 2009;324(3):962-982. https://doi.org/10.1016/j.jsv.2009.02.023.

19. Akbaş ŞD. Geometrically nonlinear static analysis of edge cracked Timoshenko beams composed of functionally graded material. Mathematical Problems in Engineering. 2013.

https://www.hindawi.com/journals/mpe/2013/87181 5/abs/. Accessed December 31, 2018.

20. Panigrahi B, Pohit G. Nonlinear modelling and dynamic analysis of cracked Timoshenko functionally graded beams based on neutral surface approach. Proceedings of the Institution of Mechanical Engineers, Part C: Journal of Mechanical Engineering Science. 2016;230(9):1486-1497. https://doi.org/10.1177/0954406215576560.

21. Yan T, Yang J, Kitipornchai S. Nonlinear dynamic response of an edge-cracked functionally graded Timoshenko beam under parametric excitation. Nonlinear Dynamics. 2012; 67(1):527-540. https://doi.org/10.1007/s11071-011-0003-9.

22. Panigrahi B, Pohit G. Study of non-linear dynamic behavior of open cracked functionally graded Timoshenko beam under forced excitation using harmonic balance method in conjunction with an iterative technique. Applied Mathematical Modelling. 2018;57:248-267.

https://doi.org/10.1016/j.apm.2018.01.022.

23. Gayen D, Tiwari R, Chakraborty D. Static and dynamic analyses of cracked functionally graded structural components: A review. Composites Part B: Engineering. 2019;173:106982.

https://doi.org/10.1016/j.compositesb.2019.106982.

24. Chajdi M, Adri A, El bikri K, Benamar R. Geometrically nonlinear free and forced vibrations analysis of clamped-clamped functionally graded beams with multicracks. MATEC Web of Conferences. 2018;211:02002. https://doi.org/10.1051/matecconf/201821102002.

25. El Kadiri M, Benamar R, White RG. Improvement of the semi-analytical method, for determining the geometrically non-linear response of thin straight structures. Part i: application to clamped-clamped and simply supported-clamped beams. Journal of Sound and Vibration. 2002; 249(2):263-305. https://doi.org/10.1006/jsvi.2001.3808.

26. Chajdi M, Fakhreddine H, Adri A, Bikri KE, Benamar R. Geometrically non-linear forced vibrations of fully 
clamped functionally graded beams with multi-cracks resting on intermediate simple supports. Journal of Physics: Conference Series. 2019; 1264:012023. https://doi.org/10.1088/1742-6596/1264/1/012023.

27. Chajdi M, Merrimi EB, El Bikri K. Geometrically nonlinear free vibration of composite materials: clamped-clamped functionally graded beam with an edge crack using homogenisation method. Key Engineering Materials. 2017; 730:521-526. https://doi.org/10.4028/www.scientific.net/KEM.730 .521.

28. Adri A, Benamar R. Linear and geometrically nonlinear frequencies and mode shapes of beams carrying a point mass at various locations. An analytical approch and a parametric study. Diagnostyka 2017; 18(2): 13-21

29. Chajdi M, Adri A, Bikri KE, Benamar R. Linear and geometrically nonlinear free and forced vibrations of fully clamped multi-cracked beams. Diagnostyka. 2019;20(1):111-125. https://doi.org/10.29354/diag/103125.

30. Fakhreddine H, Adri A, Rifai S, Benamar R. A multimode approach to geometrically non-linear forced vibrations of Euler-Bernoulli multispan beams. Journal of Vibration Engineering \& Technologies. 2020;8(2):319-326. https://doi.org/10.1007/s42417-019-00139-8.

31. Aldlemy MS, Al-jumaili S a. K, Al-Mamoori R a. M, Ya T, Alebrahim R. Composite patch reinforcement of a cracked simply-supported beam traversed by moving mass. Journal of Mechanical Engineering and Sciences. 2020;14(1):6403-6415. https://doi.org/10.15282/jmes.14.1.2020.16.0501.

32. El Bikri K, Benamar R, Bennouna MM. Geometrically non-linear free vibrations of clampedclamped beams with an edge crack. Computers \& Structures. 2006;84(7):485-502.

https://doi.org/10.1016/j.compstruc.2005.09.030.

33. Azrar L, Benamar R, White RG. A semi-analytical approach to the non-linear dynamic response problem of beams at large vibration amplitudes, part ii: multimode approach to the steady state forced periodic response. Journal of Sound and Vibration. 2002;255(1):1-41.

https://doi.org/10.1006/jsvi.2000.3595.

34. Azrar L, Benamar R, White RG. Semi-analytical approach to the non-linear dynamic response problem of $\mathrm{s}-\mathrm{s}$ and $\mathrm{c}-\mathrm{c}$ beams at large vibration amplitudes part I: general theory and application to the single mode approach to free and forced vibration analysis. Journal of Sound and Vibration. 1999; 224(2):183207. https://doi.org/10.1006/jsvi.1998.1893.

Received 2020-10-23

Accepted 2021-02-25

Available online 2021-02-26

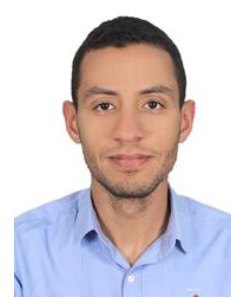

Mohcine CHAJDI - PhD student in the research team "Modeling Structures and Mechanical Systems" (M2SM) and holder of a Master's degree in Mechanical Engineering in 2015 at Higher School of Technical Education (ENSET) - Mohammed V University of Rabat, Morocco.

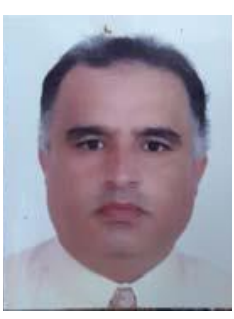

Rabat, Morocco.

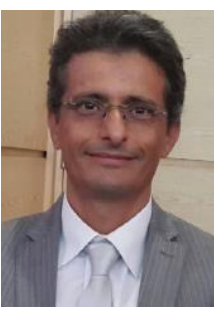

Khalid EL BIKRI - Professor of Mechanical Engineering at the Higher School of Technical Education (ENSET) - Mohammed V University of Rabat, Morocco, where he simultaneously held the post of Director. He received his Ph.D. in 2004 from the Mohammadia School of Engineering (EMI) - Mohammed V University of Rabat, Morocco. He is head of the research team "Modeling Structures and Mechanical Systems" (M2SM).

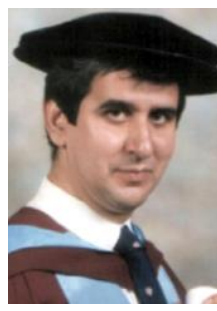

Rhali BENAMAR - Professor of Mechanical Engineering and Vibrations at the Mohammadia School of Engineering (EMI) - Mohammed V University of Rabat, Morocco. He got his engineering degree in civil engineering in 1982 from the "Ecole Nationale des Ponts et Chaussées" of Paris, France. He received his Ph.D. in 1990 from the Institute of Sound and Vibration at the University of Southampton, United Kingdom. 